\title{
A few remarks on invariable generation in infinite groups
}

\author{
Gil Goffer \\ Gennady A.Noskov \\ The Weizmann Institute of Science \\ The Sobolev Institute of Mathematics \\ 76100, Rehovot, Israel \\ 644043, Omsk, Russia
}

February 12, 2018

\begin{abstract}
A subset $S$ of a group $G$ invariably generates $G$ if $G$ is generated by $\left\{s^{g(s)} \mid s \in S\right\}$ for any choice of $g(s) \in G, s \in S$. A topological group $G$ is said to be $\mathcal{I} \mathcal{G}$ if it is invariably generated by some subset $S \subseteq G$, and $\mathcal{T} \mathcal{I} \mathcal{G}$ if it is topologically invariably generated by some subset $S \subseteq G$. In this paper we study the problem of (topological) invariable generation for linear groups and for automorphism groups of trees. Our main results show that the Lie group $\mathrm{SL}_{2}(\mathbb{R})$ and the automorphism group of a regular tree are $\mathcal{T} \mathcal{I G}$, and that the groups $P S L_{m}(K), m \geq 2$ are not $\mathcal{I} \mathcal{G}$ for countable fields of infinite transcendence degree over a prime field.
\end{abstract}

\section{Contents}

1 Introduction

2 Preliminary results

3 Parabolics and stabilizers as Wiegold subgroups

4 Borel versus Wiegold

5 Compact Lie groups and their generalizations

$6 \mathrm{SL}_{2}(\mathbb{R})$ is $\mathcal{T} \mathcal{I G}$

6.1 Conjugacy classes of $\mathrm{SL}_{2}(\mathbb{R}) \ldots \ldots \ldots \ldots \ldots \ldots \ldots$

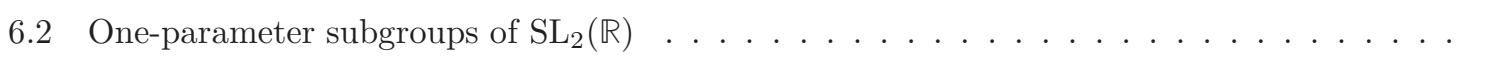

6.3 One-dimensional Lie subgroups of $\mathrm{SL}_{2}(\mathbb{R})$ and their spectra $\ldots \ldots \ldots \ldots \ldots$

6.42 -dimensional Lie subgroups of $\mathrm{SL}_{2}(\mathbb{R})$ and their spectra . . . . . . . . . . 13

6.5 Main lemma and theorem 14 . . . . . . . . . . . . . . . . . 14 
$7 \operatorname{Aut}(T)$ is $\mathcal{T} \mathcal{I G}$

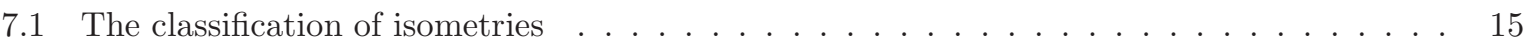

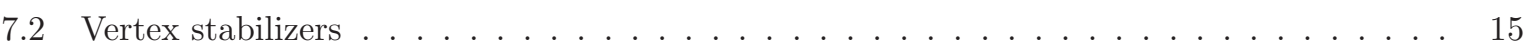

7.3 Conjugacy classes of $\operatorname{Aut}(T) \ldots \ldots \ldots \ldots \ldots \ldots \ldots \ldots$

7.4 Generating a vertex transitive subgroup $\ldots \ldots \ldots \ldots \ldots \ldots \ldots \ldots$

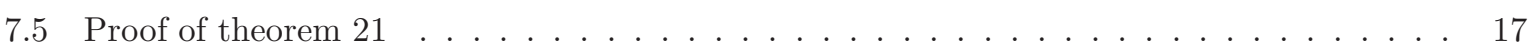

8 Uncountably many countable non- $\mathcal{I G}$-groups 18

8.1 Laws in groups $\ldots \ldots \ldots \ldots \ldots \ldots \ldots \ldots \ldots \ldots \ldots \ldots \ldots$

8.2 Rational points . . . . . . . . . . . . . . . . . . . . . . . . . . . 19

8.3 Building up free tuples . . . . . . . . . . . . . . . . . . . 19

8.4 When is $\operatorname{PSL}_{m}(K)$ an $\mathcal{I} \mathcal{G} ? \ldots \ldots \ldots \ldots \ldots \ldots$

9 Open problems

10 Acknowledgements $\quad 22$

\section{Introduction}

The notion of invariable generation of groups goes back at least as far as Jordan's paper of 1872 .

Theorem 1 ([ $[\mathbf{J o}, \mathbf{S e}])$. Assume that a finite group $G$ acts transitively on a set $X$ of cardinality at least 2. Then there exists $g \in G$ displacing every element of $X$, i.e. $g$ acts fixed-point-freely on $X$.

Independently, but more than hundred years later, J. Wiegold introduced the class $\mathfrak{X}$ of groups $G$ with the property that whenever $G$ acts transitively on a set $X$ of cardinality at least 2 , there exists an element $g \in G$ acting fixed-point-freely on $X$. (See Wi1]). Thus Jordan's theorem above says that every finite group belongs to the class $\mathfrak{X}$. Not only Wiegold failed to mention Jordan, but also he claims indirectly that Jordan's theorem is obvious. "Observe that, in particular, finite groups and soluble groups are in $\mathfrak{X}-$ obvious facts once noted, though I know of no reference to them in the literature". (See Wi1]). Wiegold gave two more characterizations of the class $\mathfrak{X}$. Namely, $G \in \mathfrak{X}$ if and only if for every proper subgroup $H \leq G$ the union $\bigcup\left\{H^{g}: g \in G\right\}$ does not cover the whole $G$. (Here, we use the common notation $H^{g}$ to denote the conjugate $g^{-1} H g$ of $H$.) Secondly, $G \in \mathfrak{X}$ if and only if every subset, containing at least one representative of every conjugacy class of $G$, generates $G$.

These characterizations lead naturally to the following definitions (of our own, though the concepts implicitly occurred in above conversation). A subset $S$ of a group $G$ is called conjugation complete (in $G$ ) if it meets every non-trivial conjugacy class of $G$. A proper subgroup $W$ of a group $G$ is called a Wiegold 
subgroup if $G$ is covered by conjugates of $W$, i.e. $G=\bigcup_{g \in G} W^{g}$. This is equivalent to saying that $W \leq G$ is proper, and $W$ is conjugation complete.

In these terms it is easy to see that the class $\mathfrak{X}$ consists of those groups that have no Wiegold subgroups, or equivalently, groups in which every conjugation complete set generates $G$. Now Jordan's theorem becomes really "obvious" for finite groups, by a counting argument. Indeed, arguing by contradiction, suppose that $G$ is a finite group with a Wiegold subgroup $W$. Then $G=\bigcup W^{g}$. There are at most $|G: W|$ members in this union, each member is of cardinality $|W|$ and all members contain the identity element, hence the cardinality of the union is strictly less than $|G: W| \cdot|W|=|G|$, contradicting that $W$ is Wiegold.

Independently of Wiegold, but somewhat later, J. D. Dixon has invented the notion of invariable generation for finite groups (see [Di]). A subset $S$ of a finite group $G$ invariably generates $G$ if $G$ is generated by $\left\{s^{g(s)} \mid s \in S\right\}$ for any choice of $g(s) \in G, s \in S$. This notion was motivated by Chebotarev's Density Theorem [Ch. W. M. Kantor, A. Lubotzky and A. Shalev, following Dixon, have defined a (not necessarily finite) group $G$ to be invariably generated if any conjugation complete subset of $G$ generates $G$ (see [KLS2]). They also discovered that the class $\mathcal{I G}$ of invariably generated groups coincides with Wiegold's class $\mathfrak{X}$. Since then a few works have been done showing that specific families of groups are invariably generated by given sets, or proving other groups are not $\mathcal{I G}$ by any subset. See the papers [Di, Ge, GM, GGJ, KLS1, KLS2, Wi1, Wi2, containing a lot of information on the class $\mathcal{I} \mathcal{G}$.

In this paper we study the notion of topological invariable generation (abbreviated to $\mathcal{T} \mathcal{I G}$ ). $\mathcal{T} \mathcal{I G}$ is a weaker property that $\mathcal{I G}$, and there are plenty of examples for groups which are $\mathcal{T} \mathcal{I G}$ but not $\mathcal{I} \mathcal{G}$. For instance, it is proved in this paper that the Lie group $\mathrm{SL}_{2}(\mathbb{R})$ is $\mathcal{T} \mathcal{I} \mathcal{G}$, whereas it seems extremely difficult to determine the status of $\mathrm{SL}_{2}(\mathbb{R})$ endowed with the discrete topology.

In this paper we study the problem of topological invariable generation for linear groups and for automorphism groups of trees. Our main results show that the Lie group $\mathrm{SL}_{2}(\mathbb{R})$ and the group of tree automorphisms $\operatorname{Aut}(T)$ are $\mathcal{T} \mathcal{I G}$ (sections 6 and 7 ), and that the groups $P S L_{m}(K), m \geq 2$ are not $\mathcal{I G}$ for countable fields of infinite transcendence degree over the prime field (section 8). In section 2 we give an alternative description of $\mathcal{T} \mathcal{I G}$ in terms of Wiegold subgroups and in terms of fixed-point free actions. We clarify the relationship between the notions of Wiegold, Borel and parabolic subgroups in sections 3-5.

\section{Preliminary results}

We shall formulate now the invariable generation problem in a topological setup. An action $G \curvearrowright X$ of a topological group $G$ on a topological space $X$ is continuous if the map $(g, x) \mapsto g x$ from $G \times X$ to $X$ is continuous. For a subgroup $W \leq G$ and an element $g \in G$, let $W^{g}=g^{-1} W g$ denote the conjugate of $W$ 
by $g$. A subset $S$ of $G$ is called conjugation complete (in $G$ ) if it meets every non-trivial conjugacy class of $G$. We call a proper closed subgroup $W$ of $G$ a Wiegold subgroup if $G$ is covered by conjugates of $W$, i.e. $G=\bigcup_{g \in G} W^{g}$, or equivalently if $W \leq G$ is proper, and $W$ is conjugation complete.

An action $G \stackrel{\alpha}{\curvearrowright} X$ of a group $G$ on a set $X$ has fixed-point property (for short, $\alpha$ is an (FP)-action) if every $g \in G$ has a fixed point in $X$. Alternatively, $\alpha$ is an FP-action if and only if $G$ is covered by the point stabilizers, i.e. $G=\bigcup_{x \in X} G_{x}$, where the stabilizer of $x$ is $G_{x}=\{g \in G: g x=x\}$.

Lemma 2 The following conditions on a topological group $G$ are equivalent:

I. $G$ contains a Wiegold subgroup.

II. There is a continuous transitive FP-action $G \curvearrowright X$ on a Hausdorff topological space $X$ of cardinality at least 2.

III. There exists a conjugation complete subset $S$ of $G$ that does not generate $G$ topologically.

\section{Proof.}

I $\Rightarrow$ II. Suppose that $G$ contains a Wiegold subgroup, say $W$. The natural action $G \curvearrowright(G / W)$ is transitive, and since $W$ is proper, the cardinality of $G / W$ is at least 2. Moreover, every $g \in G$ is contained in some conjugate $W^{h^{-1}}, h \in G$. The inclusion $g \in W^{h^{-1}}$ is equivalent to the equality $g h W=h W$, which in turn means that $g$ fixes the point $h W$ in the topological quotient $G / W$. Thus the action $G \curvearrowright(G / W)$ satisfies the fixed-point property.

II $\Rightarrow$ I. Suppose that II holds for the action $G \curvearrowright X$. We claim that the stabilizer $G_{x}$ is Wiegold for every $x \in X$. Fix $x \in X$. First, $G_{x}$ is proper since the action is transitive. Next, the stabilizer $G_{x}$ is a closed subgroup of $G$. This is because, as the action is continuous, the orbit map $\pi: G \rightarrow X: g \mapsto g x$ is continuous, and $G_{x}=\pi^{-1}(\{x\})$, with $\{x\}$ closed. By fixed-point property, $G$ is covered by stabilizers, i.e. $G=\bigcup_{y \in X} G_{y}$. And by transitivity every $y \in X$ is of the form $y=g x$ for some $g \in G$, thus $G$ is covered by stabilizers of the form $G_{g x}=g G_{x} g^{-1}$ for $g \in G$, and so $G_{x}$ is Wiegold.

I $\Rightarrow$ III. Let $W$ be a Wiegold subgroup of $G$. Then the subset $W$ of $G$ is conjugation complete, and since $W$ is a proper closed subgroup of $G$, it does not generate $G$ topologically.

III $\Rightarrow$ I. Suppose, there exists a conjugation complete subset $S$ of $G$ that does not generate $G$ topologically. The closure $W=\overline{\langle S\rangle}$ is a proper closed subgroup of $G$. Since $S$ was conjugation complete, so is $W$. 
We say that the topological group $G$ is topologically invariably generated (abbreviated to $\mathcal{T} \mathcal{I G}$ ) if the following equivalent conditions (i)-(iii) are satisfied. Note, that these conditions, being the negations of I-III, are all equivalent

(i). $\quad G$ contains no Wiegold subgroup.

(ii). If $G \curvearrowright X$ is continuous transitive action on a topological space $X$ of cardinality at least 2 then $G$ contains a fixed-point-free element.

(iii). Every conjugation complete subset of $G$ generates $G$ topologically.

In case $G$, being equipped with the discrete topology, is $\mathcal{T} \mathcal{I G}$ we say that $G$ is invariably generated (abbreviated to $\mathcal{I G}$ ). For discrete groups, conditions I-III were introduced in Wi1 and for finite groups condition III was introduced in Di]. Clearly, if $G$ is $\mathcal{I G}$ then $G$ is $\mathcal{T} \mathcal{I G}$ relative to every group topology on $G$. The converse is not true - for instance the free group $F_{2}$ of rank 2 is $\mathcal{T} \mathcal{I G}$ relative to the profinite topology but it is not $\mathcal{I G}$ (see [Wi2]).

Example 3 If $G$ is an abelian group then the conjugacy classes are just 1-element subsets of $G$. So the conjugation complete sets are those containing $G-\{1\}$. Thus every conjugation complete set generates $G$. Hence $G$ has no Wiegold subgroup and $G$ is $\mathcal{I G}$.

Example 4 Consider the extreme case when $G$ contains precisely two conjugacy classes: $\{1\}$ and $G-\{1\}$. Let us show that $G$ is $\mathcal{I G}$ if and only if $G \simeq \mathbb{Z} / 2$. Clearly, $G=\mathbb{Z} / 2$ is $\mathcal{I G}$. Conversely, suppose that $G$ is $\mathcal{I G}$. Every 1-element set $\{g\} \subset(G-\{1\})$ is conjugation complete, hence $G$ is generated by $g$ and therefore $G$ is cyclic. It is easily verified that the only cyclic group that has precisely two conjugacy classes is $\mathbb{Z} / 2$. Thus $G=\mathbb{Z} / 2$. It is proved by $\mathrm{D}$. Osin that any countable torsion free group can be embedded into a finitely generated group with exactly two conjugacy classes (see $\mathrm{Os}$ ).

These examples suggest, that intuitively the group $G$ is $\mathcal{I G}$ if it contains "many" conjugacy classes.

Lemma 5 ([Wi1, Wi2] $)$ i The class $\mathcal{I} \mathcal{G}$ is closed under extensions.

ii The class $\mathcal{I G}$ is closed under restricted direct products with arbitrarily many factors.

iii The class $\mathcal{I G}$ is not closed under unrestricted direct products and not closed under passage to subgroups.

iv The class $\mathcal{I} \mathcal{G}$ contains all finite groups and all solvable groups.

In Wi2 an $\mathcal{I} \mathcal{G}$-group is presented, whose commutator subgroup is outside $\mathcal{I} \mathcal{G}$. In the present paper it is shown that $\mathrm{SL}_{2}(\mathbb{R})$ is $\mathcal{T} \mathcal{I G}$, whereas it contains a discrete free group $F_{2}$, which is not $\mathcal{I} \mathcal{G}$.

Slightly modifying Wiegold's arguments we obtain the following properties of the class $\mathcal{T} \mathcal{I} \mathcal{G}$. 
Lemma 6 The class $\mathcal{T} \mathcal{I} \mathcal{G}$ contains all profinite groups and all topological solvable groups.

Not so many classes of groups are known to be $\mathcal{T} \mathcal{I} \mathcal{G}$ :

1. Virtually solvable groups (including finite groups) are $\mathcal{T} \mathcal{I} \mathcal{G}$ (See Wi1]).

2. The pro-finite completions of all arithmetic groups with the Congruence Subgroup Property are $\mathcal{T} \mathcal{I}$ (See $[$ KLS2] $)$.

This list is extended in the present paper by the group $\mathrm{SL}_{2}(\mathbb{R})$ and the group of automorphisms of a regular tree, which are shown to be in $\mathcal{T} \mathcal{I G}$.

The list of known non- $\mathcal{I} \mathcal{G}$ groups is lengthy but rather chaotic:

1. Groups with one single non-trivial conjugacy class, except $\mathbb{Z} / 2$.

2. The finite index net subgroups in groups $\mathrm{SO}(Q, \mathbb{Z})$ where $Q$ is a rational quadratic form of signature $(2,2)$ (see GM]).

3. All non-abelian connected compact Lie groups (see [Ge]).

4. $\mathrm{SL}_{n}(\mathbb{R})$ for $n>2$ (see [KLS2]).

5. Any non-virtually-solvable linear algebraic group over an algebraically closed field, for instance $\mathrm{SL}_{n}(\mathbb{C})$ for $n \geq 2($ see $\underline{\mathrm{KLS} 2})$.

6. Non-elementary convergence groups (including Gromov hyperbolic groups) Ge.

7. The Thompson groups $T$ and $V$ ( see GGJ]).

This list is extended in the present paper by the groups $P S L_{m}(K), m \geq 2$ where $K$ is a countable field of infinite transcendence degree over a prime field.

\section{Parabolics and stabilizers as Wiegold subgroups}

Let $\mathrm{GL}_{n}(K)$ be a general linear group of degree $n \geq 2$ over the (topological) field $K$. Consider the standard left action $\mathrm{GL}_{n}(K) \curvearrowright K^{n}$ of $\mathrm{GL}_{n}(K)$ on $K^{n}$. Let $\operatorname{Gr}(n, r)$ denote the Grassmannian of all $r$-dimensional linear subspaces of $K^{n}$. In the next proposition we give various conditions on the field and the action of a group $G$ on the Grassmannian that provide that certain stabilizers are Wiegold subgroups.

Proposition 7 Let $G$ be a subgroup of $\mathrm{GL}_{n}(K)$ and let $X \subseteq \operatorname{Gr}(n, r), n \geq r>1$ be a $G$-invariant subset with more than one element. 
1. Suppose that the action $G \curvearrowright X$ is transitive and satisfies the fixed-point property FP. Then every setwise stabilizer $G_{P}(P \in X)$ is a Wiegold subgroup of $G$.

2. In case $K$ is algebraically closed we can drop the fixed-point assumption at the expense of assuming that $X=\operatorname{Gr}(n, r)$. Namely, let $K$ be an algebraically closed field and suppose a subgroup $G \leq$ $\mathrm{GL}_{n}(K)$ acts transitively on $\operatorname{Gr}(n, r)$. Then for every $r$-dimensional linear subspace $L$ of $K^{n}$ the (setwise) stabilizer $G_{L}$ is Wiegold. In particular $\mathrm{GL}_{n}(K)$ and $\mathrm{SL}_{n}(K)$ are not $\mathcal{I} \mathcal{G}$.

3. Suppose a subgroup $G \leq \mathrm{GL}_{n}(\mathbb{R})$ acts transitively on the Grassmannian of planes $\operatorname{Gr}(n, 2)(n \geq 3)$. Then for every plane $L$ of $\mathbb{R}^{n}$ the stabilizer $G_{L}$ is Wiegold. In particular $\mathrm{GL}_{n}(\mathbb{R}), \mathrm{SL}_{n}(\mathbb{R}), \mathrm{O}_{n}(\mathbb{R})$, $\mathrm{SO}_{n}(\mathbb{R})$ are not $\mathcal{I} \mathcal{G}$.

Proof. The first assertion is just a direct consequence of lemma 2 in terms of action of $G$ on Grassmannian. The second assertion follows from the first since every $g \in \mathrm{GL}_{n}(K)$ fixes some $r$-dimensional linear subspace $L$ of $K^{n}$. (This is true for $r=1$ since $K$ is algebraically closed and for arbitrary $r$ an easy induction does the job).

As far as the third assertion is concerned, it is well known, via complexification, that every $g \in \mathrm{GL}_{n}(\mathbb{R})$ fixes a subspace of dimension 1 or 2 (see [Ax, p. 279], [PKM, Prop.12.16]). Moreover, to each nonreal eigenvalue corresponds an invariant plane. It follows that if $n \geq 2$ then every $g \in \mathrm{GL}_{n}(\mathbb{R})$ fixes a subspace of dimension 2. Then for every plane $L$ of $\mathbb{R}^{n}$ the stabilizer $G_{L}$ is Wiegold. Thus $\mathrm{GL}_{n}(\mathbb{R})$ is not $\mathcal{I} \mathcal{G}$ and

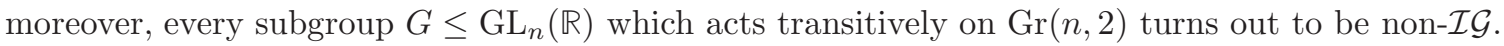

Symplectic groups. The symplectic group $S p_{2 n}(k)$ is defined as the set of all linear transformations of a $2 n$-dimensional vector space $k^{2 n}$ over the field $k$ that preserve a non-degenerate, skew-symmetric, $k$-bilinear form $\omega$ on $k^{2 n}$. A subspace $L$ of a symplectic vector space is called Lagrangian if $L=L^{\perp}$, where $L^{\perp}$ is an orthogonal complement of $L$ relative to $\omega$. A subspace $L$ is Lagrangian if and only if $\operatorname{dim} L=n$ and $L$ is isotropic, i.e. $\omega \mid L$ is identically zero. The set of Lagrangian subspaces of symplectic space $\left(k^{2 n}, \omega\right)$ is called the Lagrangian Grassmannian and is denoted by $\mathcal{L}\left(k^{2 n}\right)$. The group $S p_{2 n}(k)$ acts transitively on $\mathcal{L}\left(k^{2 n}\right)$. Indeed by Witt's theorem (1937) every linear isomorphism $f: L \rightarrow L^{\prime}$ of Lagrangian subspaces can be extended to a symplectic automorphism of $k^{2 n}$. Moreover, the following is true:

Theorem 8 ([DGHV], Theorem 3.4.3) Let $(E, \omega)$ be a symplectic vector space over a field $k$ and let $A:(E, \omega) \rightarrow(E, \omega)$ be a symplectic mapping such that all its eigenvalues are contained in $k$. Then there exists an A-invariant Lagrangian subspace $L$ of $(E, \omega)$. Moreover, if $k$ is algebraically closed then every symplectic automorphism of $(E, \omega)$ fixes (setwise) some Lagrangian subspace.

It follows that $S p_{2 n}(k)_{L}$ is Wiegold for every Lagrangian subspace $L$.

Wiegold subgroups over real closed fields. These fields have the same first-order properties as $\mathbb{R}$. A field $K$ is said to be real if -1 is not a sum of squares in $K$ (see [La, Corollary 9.3, Chapter VI]). A 
field $K$ said to be real closed if it is real, and if any algebraic extension of $K$ which is real must be equal to $K$. By a real closure we shall mean a real closed field $L$ which is algebraic over $K$. Every real field admits a real closure. If $K$ is real closed then every polynomial of odd degree in $K[X]$ has a root in $K$ and $\bar{K}:=K(\sqrt{-1})$ is the algebraic closure of $K$.

Proposition 9 Let $K$ be a real closed field. Suppose a subgroup $G \leq \mathrm{GL}_{n}(K)$ acts transitively on Grassmannian of planes $\operatorname{Gr}(n, 2)(n \geq 3)$. Then for every plane $L$ of $K^{n}$ the stabilizer $G_{L}$ is Wiegold. In particular $\mathrm{GL}_{n}(K), \mathrm{SL}_{n}(K), \mathrm{O}_{n}(K), \mathrm{SO}_{n}(K)$ are not $\mathcal{I} \mathcal{G}$.

Proof. We assert that every $\left.g \in \mathrm{GL}_{n}(K)\right)(n \geq 2)$ has invariant plane in the column space $(K(\sqrt{-1}))^{n}$. If all eigenvalues of $g$ are real, i.e. belong to $K$, then $g$ is triangulizable over $K$ and so has an invariant plane. Now suppose that there is a non-real eigenvalue $a+b \sqrt{-1} \in \bar{K},(a, b \in K) b \neq 0$. Let $w=u+\sqrt{-1} v \in \bar{K}^{n}$ be an eigenvector corresponding to $g$. Then

$$
g w=g u+\sqrt{-1} g v=(a+b \sqrt{-1})(u+\sqrt{-1} v)=(a u-b v)+\sqrt{-1}(a v+b u)
$$

from which it follows that the space $K u+K v$ is $g$-invariant. In fact this space is 2-dimensional. Indeed, suppose $u=c v, 0 \neq c \in K$, then substituting $u$ to equation 1 we obtain that $c^{2}=-1$ contradicting to assumption that $K$ is real. To complete the proof we apply now proposition 7 and obtain that the action $G \curvearrowright \operatorname{Gr}(n, 2)$ satisfies $F P$ and is transitive by assumption so $G_{L}$ is Wiegold.

In attempt to generalize proposition 9 to the subspaces of arbitrary dimension $r$ we came to

Lemma 10 Let $K$ be an algebraically closed field of degree $d \geq 2$ over its subfield $k$ and suppose that the extension $K \mid k$ is separable. Then every $g \in \mathrm{GL}_{n}(k)$ fixes a linear subspace in $k^{n}$ of dimension smaller than or equal $d$.

Proof. By Primitive Element Theorem there exists a primitive element $\zeta \in K$, i.e. $K=k(\zeta)$. It follows from finiteness assumption that $\zeta$ is algebraic over $k$. Then $K=k(\zeta)=k[\zeta]$, and $k(\zeta)$ is finite over $k$ La, Proposition 1.4., Chapter V]. Moreover, the degree $d=[k(\zeta): k]$ is equal to the degree of the irreducible polynomial $p(X)$ of $\zeta$ over $k$ (loc.cit). The proof of [La, Proposition 1.4., Chapter V] shows that the powers $1, \zeta, \ldots, \zeta^{d-1}$ form a basis for the space $K$ over $k$. Let $V=K \otimes_{k} k^{n}$ be an extension of scalars from $k$ to $K$. This is an $n$-dimensional space over $K$. The action of $g \in \mathrm{GL}_{n}(k)$ on $k^{n}$ extends naturally to an action on $K \otimes_{k} k^{n}$. Since $K$ is algebraically closed there is an eigenvector $v \in V$ with eigenvalue $\lambda \in K$. Write uniquely $\lambda=\sum_{i} l_{i} \zeta^{i}, v=\sum_{j} \zeta^{j} \otimes v_{j}$, where $l_{i} \in k, v_{i} \in k^{n}$. We assert that $g$ fixes the space $\sum_{i} k v_{i}$ (whose dimension is clearly at most $r$ ). Since $g v=\lambda v$ we have

$$
g v=\sum_{j} \zeta^{j} \otimes g v_{j}=\lambda v=\left(\sum_{i} l_{i} \zeta^{i}\right)\left(\sum_{j} \zeta^{j} \otimes v_{j}\right)=\sum_{i, j} l_{i} \zeta^{i+j} \otimes v_{j} .
$$


Rewriting $\zeta^{i+j}$ as a $k$-linear combinations of $1, \zeta, \ldots, \zeta^{r-1}$ we obtain that

$$
\sum_{i, j} l_{i} \zeta^{i+j} \otimes v_{j}=\sum_{k} \zeta^{k} \otimes v_{k}^{\prime}
$$

where $v_{k}^{\prime}$ are $k$-linear combinations of $v_{j}$. It follows from above that $g v_{j}=v_{j}^{\prime}$, and the lemma follows.

Unfortunately, the applicability of the above lemma is very much restricted by the Artin-Schreier theorem which asserts that if a field $K$ is algebraically closed with $k$ a subfield such that $1<[K: k]<\infty$ then $K=k(i)$ where $i^{2}=-1$, and $k$ has characteristic 0 [La, Corollary 9.3, Chapter VI].

\section{Borel versus Wiegold}

The following is a slight variation on the result in KLS2.

Theorem 11 Let $G$ be a linear algebraic group over an algebraically closed field $k$ and let $B$ be its Borel subgroup (=maximal connected closed solvable subgroup). 1) If $G$ is not virtually solvable then the normalizer $N_{G}(B)$ is a Wiegold subgroup of $G$. 2) If furthermore $G$ is connected then $B$ is itself Wiegold.

Proof. 1) Firstly let us prove that $N_{G}(B)$ is a proper subgroup of $G$. Suppose to the contrary that $N_{G}(B)=G$, i.e. $B$ is normal in $G$. Note that, being connected, $B$ is contained in the connected component $G_{0}$ of $G$. Since $B$ is normal in $G$, it also normal in $G_{0}$. Note that $B$ is proper in $G_{0}$ since otherwise $G$ would be a finite extension of $B=G_{0}$, i.e. virtually solvable. Finally by Chevalley result [Bo2, theorem 11.16] $B$ is self-normalizing in $G_{0}$, contradicting the normality of $B$. Hence $N_{G}(B)$ is a proper subgroup of $G$.

By a theorem of Steinberg (see Theorem 7.2 of $\underline{\mathrm{St}}$ ), every automorphism of $G$ fixes some Borel subgroup of $G$. This implies that if $g$ is any element of $G$, then $g^{-1} B^{\prime} g=B^{\prime}$ for some Borel subgroup $B^{\prime}$ of $G$. Thus the union of the normalizers $N_{G}\left(B^{\prime}\right)$ over the Borel subgroups $B^{\prime}$ of $G$ equals $G$. Since the Borel subgroups are all conjugate, it follows that $\bigcup_{g}\left(N_{G}(B)\right)^{g}=G$, thus $N_{G}(B)$ is Wiegold.

2) In this case we make use of Grothendieck's Covering Theorem [Co, Theorem 4.11]: Let $G$ be a connected linear algebraic group over algebraically closed field $k$ and let $B \leq G$ be a Borel subgroup then

$$
G(k)=\bigcup_{g \in G} g^{-1} B(k) g .
$$

This immediately implies that $B$ is Wiegold.

2-dimensional case. The standard Borel subgroup $B$ of $\mathrm{GL}_{2}(K)$ is the group of all invertible upper triangle matrices $\left(\begin{array}{ll}a & b \\ 0 & d\end{array}\right)$. For the Borel subgroup of $\mathrm{SL}_{2}(K)$ we require in addition that $a d=1$. The following exercise provides a supply of Wiegold subgroups in the 2-dimensional case, cf. [La, Exercise 23, p.547]. 
Exercise 12 Let $K$ be a field in which every quadratic polynomial has a root. Let $B$ be the Borel subgroup of $\mathrm{GL}_{2}(K)$ or of $\mathrm{SL}_{2}(K)$. Show that $B$ is Wiegold, and conclude that $\mathrm{GL}_{2}(K)$ is not $\mathcal{I} \mathcal{G}$.

Solution. We have to show that every matrix $A=\left(\begin{array}{ll}a & b \\ c & d\end{array}\right) \in \mathrm{GL}_{2}(K)$ is conjugate to a matrix in $B$. If $b=0$ we conjugate $A$ by $\left(\begin{array}{cc}0 & 1 \\ -1 & 0\end{array}\right)$. If $b \neq 0$ the conjugation $A^{X}$ by the matrix $X=\left(\begin{array}{ll}1 & 0 \\ x & 1\end{array}\right)$ gives $\left(A^{X}\right)_{2,1}=b x^{2}+(d-a) x-c$ which can be made zero by assumption. We conclude that $B$ is a Wiegold subgroup (and so $\mathrm{GL}_{2}(K)$ is not $\mathcal{I G}$ ).

An example of countable $K$ satisfying the condition of the above exercise is the "quadratic closure" of $\mathbb{Q}$ in $\mathbb{C}$. How many such fields are there?

\section{Compact Lie groups and their generalizations}

As we mentioned above every nonabelian connected compact Lie group is not $\mathcal{I} \mathcal{G}$. Here we generalize this remark to definably compact groups according to $[\mathrm{Be}]$.

We consider groups definable in an o-minimal expansion $\mathcal{M}=(R,<,+, \ldots)$ of a real closed field $(R,<,+, \ldots)$. Classical examples of such groups are the (real) algebraic subgroups of the general linear group $\mathrm{GL}_{n}(\mathbb{R})$, for instance the orthogonal group $\mathrm{SO}_{n}(\mathbb{R})$. Less classical examples of definable groups can be found in $[\mathrm{PS}$, or in $\mathrm{St}$.

Theorem 13 If $G$ is a nonabelian definably connected definably compact group in an o-minimal expansion $\mathcal{R}$ of a real closed field $R$, then for any maximal definable abelian subgroup $T$ of $G, G$ is the union of the conjugates of $T$, i.e. $T$ is a Wiegold subgroup in $G$. In particular, $G$ is not $\mathcal{I} \mathcal{G}$.

This immediately follows from the main result of $[\mathrm{Be}]$.

\section{$6 \quad \mathrm{SL}_{2}(\mathbb{R})$ is $\mathcal{T} \mathcal{I} \mathcal{G}$}

Theorem 14 The Lie group $\mathrm{SL}_{2}(\mathbb{R})$ with its canonical topology is topologically invariably generated.

Structure of the proof: The theorem will immediately follow from the key lemma 20 in which we prove that if a closed subgroup $G$ of $\mathrm{SL}_{2}(\mathbb{R})$ is conjugation complete then $G=\mathrm{SL}_{2}(\mathbb{R})$. To prove lemma 20 we show that every proper closed subgroup of $\mathrm{SL}_{2}(\mathbb{R})$ has eigen-spectrum which is strictly contained in the eigen-spectrum of $\mathrm{SL}_{2}(\mathbb{R})$. As eigen-spectrum is conjugacy invariant, a conjugation complete subgroup must have the same eigen-spectrum of $\mathrm{SL}_{2}(\mathbb{R})$, and so the lemma follows. The chapter is ordered as follows: We calculate the eigen-spectrum of $\mathrm{SL}_{2}(\mathbb{R})$ in section 6.1] Then we describe the 1-parameter subgroups of $\mathrm{SL}_{2}(\mathbb{R})$ in section 6.2 In section 6.3 we describe spectra of 1-dimensional closed subgroups of $\mathrm{SL}_{2}(\mathbb{R})$ and show that their spectra are (much) smaller than that of $\mathrm{SL}_{2}(\mathbb{R})$. In section 6.4 we do the same in case of 2-dimensional subgroups. In section 6.5 we prove the main lemma and theorem 14 
Corollary 15 The Lie group $\mathrm{PSL}_{2}(\mathbb{R})$ with its canonical topology is topologically invariably generated.

Proof. Suppose that $\mathrm{PSL}_{2}(\mathbb{R})$ admits a closed Wiegold subgroup $H$. Then the pre-image of $H$ under the quotient map $p: \mathrm{SL}_{2}(\mathbb{R}) \rightarrow \mathrm{PSL}_{2}(\mathbb{R})$ is a closed Wiegold subgroup of $\mathrm{SL}_{2}(\mathbb{R})$, contradicting Theorem 14.

\subsection{Conjugacy classes of $\mathrm{SL}_{2}(\mathbb{R})$}

Following $\left[\mathrm{Co}\right.$ we remark that conjugacy in $\mathrm{SL}_{2}(\mathbb{R})$ should not be confused with conjugacy in the larger group $\mathrm{GL}_{2}(\mathbb{R})$.

Theorem $16([\mathbf{C o}])$ Let $g \in \mathrm{SL}_{2}(\mathbb{R})$.

1. If $(\operatorname{Tr} g)^{2}>4$ then $g$ is conjugate to a unique matrix of the form

$$
\left(\begin{array}{cc}
\lambda & 0 \\
0 & 1 / \lambda
\end{array}\right)
$$

with $\lambda \in \mathbb{R},|\lambda|>1$.

2. If $(\operatorname{Tr} g)^{2}=4$ then $g$ is conjugate to exactly one of

$$
\pm I_{2}, \pm\left(\begin{array}{ll}
1 & 1 \\
0 & 1
\end{array}\right), \pm\left(\begin{array}{cc}
-1 & 1 \\
0 & -1
\end{array}\right)
$$

3. If $(\operatorname{Tr} g)^{2}<4$ then $g$ is conjugate to a unique matrix of the form

$$
\left(\begin{array}{cc}
\cos \theta & -\sin \theta \\
\sin \theta & \cos \theta
\end{array}\right)
$$

other than $\pm I_{2}$.

For a set of (complex) matrices $M$ let $\operatorname{Sp}(M)$ to denote the eigenvalue spectrum of $M$, i.e. the set of all eigenvalues of matrices in $M$ (counting without multiplicity). The following is an immediate consequence of the above result.

Corollary $17 \mathrm{Sp}\left(\mathrm{SL}_{2}(\mathbb{R})\right)=\mathbb{R}^{\times} \cup \mathbb{S}$, where $\mathbb{R}^{\times}=\mathbb{R}-\{0\}$ and $\mathbb{S}=\{z \in \mathbb{C}:|z|=1\}$.

\subsection{One-parameter subgroups of $\mathrm{SL}_{2}(\mathbb{R})$}

Recall that a one-parameter subgroup in $\mathrm{GL}_{n}(\mathbb{R})$ is a homomorphism $\mathbb{R} \rightarrow \mathrm{GL}_{n}(\mathbb{R})$ and it is necessarily of the form $f(t)=e^{A t}$ where $A$ is the matrix $f^{\prime}(0)$. We describe here the conjugacy classes of one-parameter subgroups in $\mathrm{SL}_{2}(\mathbb{R})$. For that we need to know the orbits of $\mathrm{SL}_{2}(\mathbb{R})$-action on its Lie algebra. We set

$$
\mathfrak{s l}_{2}(\mathbb{R})=\left\{X \in M_{2}(\mathbb{R}) \mid \operatorname{Tr} X=0\right\}=\left\{\left(\begin{array}{cc}
a & b \\
c & -a
\end{array}\right): a, b, c \in \mathbb{R}\right\}
$$


to denote the Lie algebra of the Lie group $\mathrm{SL}_{2}(\mathbb{R})$. For a nonzero $2 \times 2$ real matrix $X=\left(\begin{array}{cc}a & b \\ c & -a\end{array}\right) \in \mathfrak{s l}_{2}(\mathbb{R})$ we have that the characteristic polynomial is $x^{2}-\left(a^{2}+b c\right)$ and therefore its eigenvalues are $\pm \sqrt{a^{2}+b c}$. There are three cases:

1) $a^{2}+b c>0$. In this case both eigenvalues are real and non-zero. Denote them by $\pm t$. Then $X$ is conjugate by some $g \in \mathrm{GL}_{2}(\mathbb{R})$ to the diagonal matrix $\left(\begin{array}{cc}t & 0 \\ 0 & -t\end{array}\right.$. $)$. To see that $X$ is also conjugate to $\left(\begin{array}{cc}t & 0 \\ 0 & -t\end{array}\right)$ by the smaller group $\mathrm{SL}_{2}(\mathbb{R})$ we replace $g$ by $\left(\begin{array}{cc}\operatorname{det}(g)^{-1} & 0 \\ 0 & 1\end{array}\right) g$.

Matrices of the form $\left(\begin{array}{cc}t & 0 \\ 0 & -t\end{array}\right)$ constitute a 1-dimensional Lie subalgebra $\mathfrak{a}=\mathbb{R}\left(\begin{array}{cc}1 & 0 \\ 0 & -1\end{array}\right)$ of $\mathfrak{s l}_{2}(\mathbb{R})$ called the split Cartan subalgebra. Exponentiating elements of $\mathfrak{a}$ we obtain the split Cartan subgroup

$$
A=\left\{\left(\begin{array}{cc}
e^{t} & 0 \\
0 & e^{-t}
\end{array}\right): t \in \mathbb{R}\right\},
$$

which is a 1-parameter subgroup of $\mathrm{SL}_{2}(\mathbb{R})$ corresponding to subalgebra $\mathfrak{a}$.

2) $a^{2}+b c=0$. In this case $X$ has zero eigenvalue of multiplicity 2 . Then $X$ is conjugate by some $g \in \mathrm{GL}_{2}(\mathbb{R})$ to a nilpotent matrix of the form $\left(\begin{array}{ll}0 & d \\ 0 & 0\end{array}\right), 0 \neq d \in \mathbb{R}$. Again we can make $g$ to be in $\mathrm{SL}_{2}(\mathbb{R})$ replacing $g$ by the matrix $\left(\begin{array}{cc}\operatorname{det}(g)^{-1} & 0 \\ 0 & 1\end{array}\right) g$.

Matrices of the form $\left(\begin{array}{ll}0 & t \\ 0 & 0\end{array}\right)$ constitute a 1-dimensional Lie subalgebra $\mathfrak{n}=\mathbb{R}\left(\begin{array}{ll}0 & 1 \\ 0 & 0\end{array}\right)$ of $\mathfrak{s l}_{2}(\mathbb{R})$ called nilpotent subalgebra $\mathfrak{n}$. Exponentiating elements of $\mathfrak{n}$ we obtain the unipotent subgroup

$$
U=\left(\begin{array}{ll}
1 & \mathbb{R} \\
0 & 1
\end{array}\right)
$$

which is a 1-parameter subgroup of $\mathrm{SL}_{2}(\mathbb{R})$ corresponding to the subalgebra $\mathfrak{n}$.

3) $a^{2}+b c<0$. In this case $X$ has nonzero purely imaginary conjugate eigenvalues $\pm \theta i \quad(\theta=$ $\left.\sqrt{-a^{2}-b c}\right)$. We claim that $X$ is conjugate to one of the matrices $\pm \theta\left(\begin{array}{cc}0 & 1 \\ -1 & 0\end{array}\right)$ by a matrix from $\mathrm{SL}_{2}(\mathbb{R})$. There are real linearly independent column vectors $u, v$ such that $X(u+i v)=\theta i(u+i v)=\theta(-v+i u)=$ $X u+i X v$, from what we obtain $X u=-\theta v, X v=\theta u$. It follows that a 2-by-2 matrix $(u, v) \in M_{2}(\mathbb{R})$ with columns $u, v$ is invertible. We have $X(u, v)=(X u, X v)=\theta(-v, u)=\theta(u, v)\left(\begin{array}{cc}0 & 1 \\ -1 & 0\end{array}\right)$.

It follows that

$$
(u, v)^{-1} X(u, v)=\theta\left(\begin{array}{cc}
0 & 1 \\
-1 & 0
\end{array}\right) .
$$

We wish to modify $g=(u, v)$ to be in $\mathrm{SL}_{2}(\mathbb{R})$. By multiplying the matrix $g$ by $\sqrt{|g|}^{-1}$, we may assume $\operatorname{det}(g)= \pm 1$. If $\operatorname{det}(g)=-1$ then we replace $g$ by $g\left(\begin{array}{cc}1 & 0 \\ 0 & -1\end{array}\right)$, which is in $\mathrm{SL}_{2}(\mathbb{R})$, and we get that $g^{-1} X g=-\theta g\left(\begin{array}{cc}0 & 1 \\ -1 & 0\end{array}\right)$.

Matrices of the form $\left(\begin{array}{cc}0 & t \\ -t & 0\end{array}\right)$ constitute a 1-dimensional Lie subalgebra $\mathfrak{k}=\mathbb{R}\left(\begin{array}{cc}0 & 1 \\ -1 & 0\end{array}\right)$ of $\mathfrak{s} \mathfrak{s}_{2}(\mathbb{R})$ called the non-split Cartan subalgebra. Exponentiating elements of $\mathfrak{k}$ we obtain a 1-parameter subgroup $K=\mathrm{SO}_{2}(\mathbb{R})$ of rotations called the non-split Cartan subgroup: 


$$
K=\left\{\left(\begin{array}{cc}
\cos t & \sin t \\
-\sin t & \cos t
\end{array}\right): t \in \mathbb{R}\right\} .
$$

Clearly $\mathrm{SO}_{2}(\mathbb{R})$ is a compact subgroup of $\mathrm{SL}_{2}(\mathbb{R})$. In fact, it is a maximal closed subgroup of $\mathrm{SL}_{2}(\mathbb{R})$ Gl, Ch.VI, section 1].

\subsection{One-dimensional Lie subgroups of $\mathrm{SL}_{2}(\mathbb{R})$ and their spectra}

Lemma 18 The eigen-spectrum of every closed 1-dimensional subgroup in $\mathrm{SL}_{2}(\mathbb{R})$ is a proper subset in $\mathrm{Sp}\left(\mathrm{SL}_{2}(\mathbb{R})\right)$.

\section{Proof.}

Suppose that $G$ is a Lie subgroup of $\mathrm{SL}_{2}(\mathbb{R})$ such that its component $G^{0}$ is 1-dimensional, so is a 1-parameter subgroup of one of the above types $A, U, K$.

1) In case $G^{0}=A$ we have

$$
A=G^{0} \unlhd G \unlhd N(A),
$$

where $N(A)$ is the normalizer of $A$ in $\mathrm{SL}_{2}(\mathbb{R})$. By Bruhat decomposition $N(A)=A \cup w A$, where $w=\left(\begin{array}{cc}0 & 1 \\ -1 & 0\end{array}\right)$ is the Weyl element (see [La, p.539]). We conclude that

$$
\operatorname{Sp}(G) \leq \operatorname{Sp}(N(A))=\operatorname{Sp}(A) \cup \operatorname{Sp}(w A) .
$$

Note that (surprisingly but luckily) $\operatorname{Sp}(w A)$ equals

$$
\operatorname{Sp}\left\{\left(\begin{array}{cc}
0 & e^{-t} \\
-e^{t} & 0
\end{array}\right), t \in \mathbb{R}\right\}=\{ \pm i\}
$$

and indeed $\operatorname{Sp}(G) \leq \mathbb{R}^{\times} \cup\{ \pm i\}$ is a proper subset of $\operatorname{Sp}\left(\mathrm{SL}_{2}(\mathbb{R})\right)$.

2) If $G^{0}=U$ is unipotent, then it is easy to see $N(U)=B$, where $B=\left\{ \pm\left(\begin{array}{cc}e^{t} & s \\ 0 & e^{-t}\end{array}\right) ; t, s \in R\right\}$. Hence $G^{0} \unlhd G \unlhd N(U)=B$ and clearly $\operatorname{Sp}(G) \leq \operatorname{Sp}(B)=\mathbb{R}^{\times}$.

3) Finally, in case $G^{0}=K$ we have $N(K)=K$, see [Gl, Ch.VI, sec. 1], hence $\operatorname{Sp}(G) \leq \operatorname{Sp}(K)=\mathbb{S}$.

\subsection{2-dimensional Lie subgroups of $\mathrm{SL}_{2}(\mathbb{R})$ and their spectra}

A two-dimensional Lie algebra over $\mathbb{R}$ is either abelian or solvable. Furthermore a solvable Lie algebra $\mathfrak{s}_{2}$ has generators $e, f$ and defining relation $[e f]=e$ ( see [Ja $)$. Using the above classification of 1dimensional subalgebras it is easy to conclude that there are no two-dimensional abelian subalgebras in 
$\mathfrak{s l}_{2}(\mathbb{R})$. On the other hand there is only one (up to conjugacy) subalgebra isomorphic to $\mathfrak{s}_{2}$, namely the Borel subalgebra

$$
\mathfrak{b}=\left\{\left(\begin{array}{cc}
t & s \\
0 & -t
\end{array}\right) ; t, s \in \mathbb{R}\right\} .
$$

The (connected) component $B^{0}$ of the Borel subgroup

$$
B=\left\{ \pm\left(\begin{array}{cc}
e^{t} & s \\
0 & e^{-t}
\end{array}\right) ; t, s \in R\right\}
$$

has $\mathfrak{b}$ as its Lie algebra. It follows that $B^{0}$ is the only (up to conjugacy) 2-dimensional connected subgroup of $\mathrm{SL}_{2}(\mathbb{R})$. We conclude with

Lemma 19 Let $G$ be a Lie subgroup of $\mathrm{SL}_{2}(\mathbb{R})$ such that its component $G^{0}$ is a component $B^{0}$ of the Borel subgroup $B$. Then $G \leq B$ and $\operatorname{Sp}(G)=\mathbb{R}^{\times}$.

\subsection{Main lemma and theorem 14}

Lemma 20 If a closed subgroup $G$ of $\mathrm{SL}_{2}(\mathbb{R})$ is conjugation complete then $G=\mathrm{SL}_{2}(\mathbb{R})$.

Proof. Suppose, on the contrary, that there exists a proper closed and conjugation complete subgroup $G$ in $\mathrm{SL}_{2}(\mathbb{R})$. It follows from conjugation completeness that $\operatorname{Sp}(G)=\operatorname{Sp}\left(\mathrm{SL}_{2}(\mathbb{R})\right)$.

By Cartan's theorem any closed subgroup of $\mathrm{GL}_{n}(\mathbb{R})$ is a Lie group. (A short elegant proof can be found in $\underline{\mathrm{Ad}}$, pages 17-19). Hence $G$ is a Lie subgroup of $\mathrm{SL}_{2}(\mathbb{R})$. The connected component $G^{0}$ of $G$ is a proper closed normal Lie subgroup of $G$. Since $G$ is a proper subgroup of 3-dimensional connected Lie group $\mathrm{SL}_{2}(\mathbb{R})$, we conclude that $\operatorname{dim} G^{0}=0,1$ or 2 .

If $\operatorname{dim} G^{0}=0$ then $G$ is at most countable discrete subgroup of $\mathrm{SL}_{2}(\mathbb{R})$ and thus its spectrum is at most countable, so $\operatorname{Sp}(G)$ is properly contained in $\mathbb{R}^{\times} \cup \mathbb{S}=\operatorname{Sp}\left(\mathrm{SL}_{2}(\mathbb{R})\right)$. Contradiction.

If $\operatorname{dim} G^{0}=1$ then $G^{0}$ is a 1-parameter subgroup of $\mathrm{SL}_{2}(\mathbb{R})$ and by lemma 18 the eigen-spectrum of $G$ is a proper subset in $\mathrm{Sp}\left(\mathrm{SL}_{2}(\mathbb{R})\right)$. Contradiction.

Finally if $\operatorname{dim} G^{0}=2$ then $G^{0}$ is the connected component of the Borel subgroup and by lemma 19 the eigen-spectrum of $G$ is a proper subset in $\operatorname{Sp}\left(\mathrm{SL}_{2}(\mathbb{R})\right)$. Contradiction.

The lemma and thereby theorem 14 are proved.

\section{$7 \quad \operatorname{Aut}(T)$ is $\mathcal{T} \mathcal{I G}$}

A (simplicial) graph $\Gamma=(V, E)$ consists of a set of vertices $V$ and a family $E$ of edges which are 2element subsets of $V$. A (simple) path from vertex $x$ to vertex $y$ is a sequence of vertices $\pi: x=$ $x_{0}, x_{1}, \ldots, \ldots, x_{n}=y$ such that every subset $\left\{x_{i}, x_{i+1}\right\}$ is an edge and every two subsequent edges are 
distinct. The number $n \geq 0$ is the length of $\pi$. Define the distance $d(x, y)$ between vertices $x$ and $y$ to be the minimum of the lengths of simple paths joining them. A loop is a path of positive length such that $x_{0}=x_{n}$. A tree is a (simplicial) graph without loops. The valence or degree of a vertex $x$ in a tree $T$ is the number of edges that contain $x$. A regular $d$-tree is a tree with every vertex having degree $d$. Consider the group $\operatorname{Aut}(T)$ of automorphisms (=isometries) of $T$, equipped with the standard pointwise convergence topology. Then $\operatorname{Aut}(T)$ turns into a totally disconnected, locally compact, unimodular topological group. In this section we prove the following theorem:

Theorem 21 The automorphism group $\operatorname{Aut}(T)$ of a d-regular tree is $\mathcal{T} \mathcal{I} \mathcal{G}$ for every $d \geq 2$.

\subsection{The classification of isometries}

According to Ti for a single automorphism $g$ of a $d$-regular tree $T$ there are three mutually exclusive cases to consider:

- $g$ is elliptic, i.e. $g$ has a fixed point in $V(T)$,

- $g$ is an inversion, i.e. there is an edge which is flipped by $g$,

- $g$ is hyperbolic with an axis $A_{g}$ : that is to say $A_{g}$ is a subtree with all vertices of degree 2 and $g$ acts on $A_{g}$ by translation by a positive amount, denoted $|g|$. The number $|g|$ is called the translation length of $g$.

We will denote by $\mathscr{E}, \mathscr{I}, \mathscr{H}$ the classes of elliptics, inversions and hyperbolics, respectively. It is easy to see that they are all nonempty and pairwise disjoint. Moreover each of these classes is invariant under conjugation.

Observe that conjugation translates the axes and the fixed points, i.e. if $g, h \in \operatorname{Aut}(T)$ and $h$ is hyperbolic then $A_{g^{-1} h g}=g A_{h}$. If $h$ is elliptic fixing $v \in V(T)$, then $g^{-1} h g$ fixes $g v$, and if $h$ is an inversion flipping the edge $e \in E(T)$, then $g^{-1} h g$ flips $g e$.

\subsection{Vertex stabilizers}

Let $T$ be a $d$-regular tree, $d \geq 2$. We denote the pointwise stabilizer in $\operatorname{Aut}(T)$ of a subset $M \subset V(T)$ by $\operatorname{Stab}_{\mathrm{Aut}(T)}(M)$, or by $\operatorname{Stab}(M)$. When $v \in V(T)$, we may simply write $\operatorname{Stab}(v)$ to mean $\operatorname{Stab}(\{v\})$. When $K \leq \operatorname{Aut}(T)$ is a subgroup, we denote by $\operatorname{Stab}_{K}(M)$ the stabilizer of $M$ inside $K$. For a vertex $v \in V(T)$ and $n \geq 0$ we denote by $B(v, n) \subset V(T)$ the closed ball of radius $n$ about $v$.

Fix a vertex $v \in V(T)$ and denote by $(T, v)$ the rooted tree $T$ with a root $v$. We identify the stabilizer subgroup $\operatorname{Stab}(v) \leq \operatorname{Aut}(T)$ with the group $\operatorname{Aut}(T, v)$ of automorphisms of the rooted tree $(T, v)$. The group $\operatorname{Aut}(T, v)$ is a profinite group. Indeed, it is the inverse limit of its finite quotients 
$\operatorname{Aut}(T, v) / \operatorname{Stab}(B(v, n))$. Just like finite groups, every profinite group is $\mathcal{T} \mathcal{I} \mathcal{G}$. The proof is due to KLS2, we sketch it here for completeness:

Lemma 22 A profinite group is $\mathcal{T} \mathcal{I} \mathcal{G}$.

Proof.(sketched). Let $G$ be a profinite group. Every proper closed subgroup of a profinite group is contained in a maximal open subgroup $M$. Since $M$ has finite index it cannot be conjugation complete, hence G contains no Wiegold subgroups.

Corollary 23 The group Aut $(T, v)$ of automorphisms of a rooted d-regular tree, $d \geq 2$, is $\mathcal{T} \mathcal{I} \mathcal{G}$.

In the next section we present the conjugacy classes of $\operatorname{Aut}(T)$ and observe the relations between conjugacy in $\operatorname{Aut}(T)$ and conjugacy in $\operatorname{Aut}(T, v)$.

\subsection{Conjugacy classes of Aut $(T)$}

We follow GNS for the description of the conjugacy classes of the groups Aut $(T)$ and $\operatorname{Aut}(T, v)$. For $g \in \operatorname{Aut}(T)$ we define the orbital type of $g$ to be the labeled graph $T_{g}$ of $g^{\mathbb{Z}}$-orbits, where $g^{\mathbb{Z}}$ is the cyclic group generated by $g$. That is, the vertices of $T_{g}$ are $g^{\mathbb{Z}}$-orbits; An edge $\left(o_{1}, o_{2}\right) \in E\left(T_{g}\right)$ is connecting two orbits $o_{1}, o_{2} \in V\left(T_{g}\right)$ if the orbits contain vertices $x_{1} \in o_{1}, x_{2} \in o_{2}$ which were connected by an edge $\left(x_{1}, x_{2}\right) \in E(T)$ in the original tree; Each $g^{\mathbb{Z}}$-orbit is labeled by its cardinality, in $\mathbb{N} \cup\{\infty\}$. Two orbital types are called equivalent if they are isomorphic as labeled trees. We call an orbital type elliptic if it contains an orbit labeled by 1 . Observe that $g$ has an elliptic orbital type if and only if $g$ is elliptic.

Any conjugate of an elliptic element (inversion, hyperbolic) is clearly elliptic (resp. inversion, hyperbolic) as well. Therefore, conjugacy classes of $\operatorname{Aut}(T)$ can be described separately in each of these cases.

Lemma 24 If $g, g^{\prime} \in \operatorname{Aut}(T)$ are both elliptic or both inversions then they are conjugate to each other if and only if their orbital types are equivalent. If $h, h^{\prime} \in \operatorname{Aut}(T)$ are hyperbolic then they conjugate to each other if and only if they have equal translation length.

In the rooted tree $(T, v)$, all elements are elliptic. We have therefore the following characterization:

Lemma 25 Elements $g, g^{\prime} \in A u t(T, v)$ are conjugate to each other (in Aut $(T, v)$ ) if and only if their orbital types are equivalent.

For the full proofs of lemma 24 and lemma 25 see GNS.

Denote by $\mathscr{O}$ the collection of all elliptic orbital types of elements in $\operatorname{Aut}(T)$. A direct corollary is the following: 
Corollary 26 The collection of all orbital types of elements in Aut $(T, v)$ is $\mathscr{O}$.

Proof. Clearly the collection of all orbital types of elements in $A u t(T, v)$ is contained in $\mathscr{O}$, as $A u t(T, v)=$ $S t a b_{\text {Aut }}(T)(v)$ is a subgroup of Aut $(T)$. For the other inclusion observe that every element $g$ in Aut $(T)$ which has an elliptic orbital type is elliptic, therefore stabilizes some $u \in V(T)$. Let $h \in \operatorname{Aut}(T)$ be an element such that $h . u=v$, then $h g h^{-1}$ is in $\operatorname{Stab}(v)$ and has the same orbital type as $g$.

\subsection{Generating a vertex transitive subgroup}

Let $s \in \operatorname{Aut}(T)$ be an elliptic element stabilizing a vertex $v \in V(T)$ and assume that the action of $s^{\mathbb{Z}}$ is transitive on all spheres about $v$. We call such an element $v$-spherically transitive. An elliptic element $s \in \operatorname{Aut}(T)$ is called spherically transitive if it is $v$-spherically transitive for some $v$. For any $v \in V(T)$ there exists a $v$-spherically transitive element in $\operatorname{Aut}(T)$ (see [BORT). If $s$ is $v$-spherically transitive, then the orbital type of $s$ is an infinite ray $\left[v=x_{0}, x_{1}, x_{2}, \ldots\right)$ with labels $f\left(x_{0}\right)=1, f\left(x_{n}\right)=d(d-1)^{n-1}$, $n \geq 1$ (that is, the size of the $n^{t h}$-sphere about $v$ ). Hence, all spherically transitive elements in Aut $(T)$ are conjugate.

Lemma 27 Let $h$ be a hyperbolic automorphism of translation length $|h|=1$ and let $s$ be a spherically transitive element. Then $\langle h, s\rangle$ acts transitively on the vertices of $T$.

Proof. Let $v$ denote the fixed point of $s, A_{h}$ denote the axis along which $h$ translates and $m$ denote the distance between $v$ and $A_{h}$ (possibly $m=0$ ). Since $h$ acts transitively on the vertices of $A_{h}$, it is enough to show that any vertex in $T$ can be mapped by $\langle h, s\rangle$ into $A_{h}$. Indeed, let $y \in V(T)$ be a vertex. For large enough $k$ we have that $d\left(h^{k} . y, v\right)>m$. Since every sphere of radius at least $m$ around $v$ intersects $A_{h}$, we have that some element in $\langle s\rangle$ maps $h^{k} . y$ into $A_{h}$, and so the lemma is proved.

The following is a direct corollary from the last lemma and the structure of conjugacy classes in $\operatorname{Aut}(T)$.

Corollary 28 every conjugation complete subset of Aut $(T)$ generates a vertex transitive subgroup.

Proof. Such a set must contain hyperbolic elements of all translation lengths $l \in\{1,2, \ldots\}$ and elliptic elements of all orbital types $i \in \mathscr{O}$. In particular it must contain a hyperbolic element of translation length 1 and a spherically transitive element, therefore it satisfies the assumptions of lemma 27

\subsection{Proof of theorem 21}

We prove the following rephrasement of theorem 21.

Theorem 29 Every closed conjugation complete subgroup $H$ of the automorphism group $\operatorname{Aut}(T)$ of an $m$-regular tree $T,(m \geq 2)$, coincides with $\operatorname{Aut}(T)$. 
Proof. Let $H \leq \operatorname{Aut}(T)$ be a closed conjugation complete subgroup. By corollary $28 H$ is vertextransitive. Furthermore, since $H$ is conjugation complete, it contains elements of all elliptic orbital types. Let $\left\{r_{i}\right\}_{i \in \mathscr{O}} \subseteq H$ be a set of representatives in $H$ for all orbital types $i \in \mathscr{O}$. Each $r_{i}$ is elliptic, and has a non-empty set of fixed points in $V(T)$. For every $i \in \mathscr{O}$ let $v_{i}$ be a fixed point of $r_{i}$. Fix $v \in V(T)$ and denote by $K=\operatorname{Stab}_{\operatorname{Aut}(T)}(v)$. Since $H$ is vertex transitive, we can find elements $h_{i} \in H$ s.t $h_{i} \cdot v_{i}=v$ for all $i$. Consider the collection $\mathcal{C}=\left\{h_{i} r_{i} h_{i}^{-1}\right\}_{i \in \mathscr{O}} \subseteq H$. As conjugation does not affect the orbital type, this collection contains elements of all orbital types in $\mathscr{O}$. Further, all elements in $\mathcal{C}$ stabilize $v$. So $\mathcal{C}$ is a conjugation complete collection in $K$, with respect to conjugacy inside $K$. By corollary 23 $K=\operatorname{Aut}(T, v)$ is $\mathcal{T} \mathcal{I} \mathcal{G}$, so in particular, the collection $\mathcal{C} \subseteq H$ generates $K$, which implies that $K \leq H$.

We obtain that $H$ is vertex transitive and contains the vertex-stabilizer group $K=\operatorname{Stab}_{\operatorname{Aut}(T)}(v)$. It follows that $H=\operatorname{Aut}(T)$.

\section{Uncountably many countable non- $\mathcal{I} \mathcal{G}$-groups}

\subsection{Laws in groups}

A non-empty word $w=w\left(x_{1}, \ldots, x_{n}\right)$ (= string of symbols in alphabet $\left.x_{1}^{ \pm 1}, \ldots, x_{n}^{ \pm 1}\right)$ is reduced if it does not contain adjacent symbols of the form $x_{i}^{\varepsilon} x_{i}^{-\varepsilon}, \varepsilon= \pm 1$. A non-empty word $w=w\left(x_{1}, \ldots, x_{n}\right)$ is reduced on the tuple of elements $\left(g_{1}, \ldots, g_{n}\right)$ of a group $G$ if $w$ is reduced and every word of the form $x_{i}^{m}$ does not occur as a subword in $w$ when $|m| \geq\left|g_{i}\right|$, where $\left|g_{i}\right|$ denotes the order of $g_{i}$ ( we of course assume that $\left|g_{i}\right|=\infty$ when $g_{i}^{\mathbb{Z}}$ is infinite cyclic). A tuple of elements $\left(g_{1}, \ldots, g_{n}\right)$ of a group $G$ is free if the group $\left\langle g_{1}, \ldots, g_{n}\right\rangle$ generated by them is a free product of cyclics $g_{i}^{\mathbb{Z}}$, i.e. the group $\left\langle g_{1}, \ldots, g_{n}\right\rangle$, generated by $g_{1}, \ldots, g_{n}$, is isomorphic to the free product $g_{1}^{\mathbb{Z}} * \cdots * g_{n}^{\mathbb{Z}}$ under the natural homomorphism from $g_{1}^{\mathbb{Z}} * \cdots * g_{n}^{\mathbb{Z}}$ to $\left\langle g_{1}, \ldots, g_{n}\right\rangle$. A word $w$ is said to be a law in a group $G$ if $w$ becomes trivial whatever values the arguments $x_{i}$ are assigned from $G$; i.e. $w\left(g_{1}, \ldots, g_{n}\right)=1$ for all $g_{i} \in G$.

The proof of the following lemma is routine and is left to the reader.

Lemma 30 A tuple $\left(g_{1}, \ldots, g_{n}\right)$ over a group $G$ is free if and only if every non-empty word $w\left(x_{1}^{ \pm 1}, \ldots, x_{n}^{ \pm 1}\right)$, which is reduced over the tuple $\left(g_{1}, \ldots, g_{n}\right)$, does not vanish on $\left(g_{1}, \ldots, g_{n}\right)$, i.e. $w\left(g_{1}, \ldots, g_{n}\right) \neq e$. In other words a tuple $\left(g_{1}, \ldots, g_{n}\right)$ is free if and only if there are no non-trivial relations between the elements $g_{1}, \ldots, g_{n}$.

More generally, consider a free product $G[x]=G * x^{\mathbb{Z}}$ of $G$ and the infinite cyclic group $x^{\mathbb{Z}}$. An element $w \in G[x]$ will be called a monomial in variable $x$ over the group $G$. In case $w \notin G$ we say that $w$ is non-constant. A monomial $w$ induces a map $w: G \rightarrow G$, where $w(g)$ is the image of $w$ under the natural group homomorphism

$$
G[x] \rightarrow G
$$


taking $x$ to $g$ and fixing $G$ pointwise. The subset $V(w)=\{(g \in G: w(g)=1\}$ is called the principal algebraic set of $G$ corresponding to $w$. Every monomial $w \in G[x]$ can be written as a word $w=a_{0} x^{l_{1}} a_{1} x^{l_{2}} \cdots a_{m-1} x^{l_{m}} a_{m}$ with $a_{i}$-s in $G$. We say that this word expression is reduced over $G$ if it does not contain a subword $x^{ \pm 1} a x^{\mp 1}$ with $a$ in the center of $G$. If $a$ is central then replacing $x^{ \pm 1} a x^{\mp 1}$ by $a$ does not change the principal algebraic set $V(w)$. After finitely many such replacements any word $w$ can be brought to a reduced form (which, of course, could occur to be empty or constant). A (non-empty) reduced non-constant word $w(x)=a_{0} x^{l_{1}} a_{1} x^{l_{2}} \cdots a_{m-1} x^{l_{m}} a_{m}$ over $G$ is said to be a generalized law in a group $G$ if $w$ becomes trivial whatever values the arguments $x$ are assigned from $G$; i.e. $w(g)=1$ for all $g \in G$.

The following is the simplified version of the result by Mikhalev and Golubchik, showing that there is no generalized law in $\mathrm{GL}_{m}(K),(m \geq 2)$ in case of infinite field $K$.

Theorem 31 ([GM] $)$ Let $K$ be an infinite field and $w(x)=g_{0} x^{i_{0}} g_{1} x^{i_{1}} \cdots g_{m} x^{i_{m}} g_{m+1}$ be a monomial over the group $\mathrm{GL}_{m}(K),(m \geq 2)$. If $g_{1}, \ldots, g_{m}$ are all non-central in $\mathrm{GL}_{m}(K)$ then the principal algebraic set $V(w)=\left\{g \in G L_{m}(K): w(g)=1\right\}$ is a proper subset of $G L_{m}(K)$.

The same result (with the same proof) is true for the groups $\mathrm{SL}_{m}(K)$ and $\mathrm{PSL}_{m}(K)$.

\subsection{Rational points}

As in Bo1 we identify implicitly an algebraic group $G$ with the group $G(\Omega)$ of its points in a "universal domain" $\Omega$, i.e. an algebraically closed extension of infinite transcendence degree over a prime field (recall that the prime field is either the rational number field $\mathbb{Q}$, or a finite field of prime order $\mathbb{F}_{p}$ ).

Assume now that $G$ is defined over a field $K$ of infinite transcendence degree over a prime field $k$. We shall need the following lemma which guaranties that, under some natural conditions, given a family of proper algebraic subsets $V_{i}(i \in \mathbb{N})$ of $G$, the set of $K$-rational points outside of a countable union $\cup V_{i}(i \in \mathbb{N})$ is nonempty. (This is kind of an algebraic analog of Baire category theorem).

Lemma 32 ([Bo1], §2,Lemma 2) Let $K$ be a field of infinite transcendence degree over a prime field. Let $X$ be an irreducible unirational $K$-variety. Let $L$ be a finitely generated subfield of $K$ containing a field of definition of $X$, and $V_{i}(i \in \mathbb{N})$ a family of proper algebraic subsets of $X$ defined over an algebraic closure $\bar{L}$ of $L$. Then $X(K)$ is not contained in the union of the $V_{i}(i \in \mathbb{N})$.

\subsection{Building up free tuples}

Lemma 33 (main) Let $K$ be a field of infinite transcendence degree over a prime field $k$. Let $C_{1}, \ldots, C_{n}$ be non-trivial distinct conjugacy classes of the group $G=\mathrm{PSL}_{m}(K)$, where $n, m \geq 2$. Suppose that 
$c_{1} \in C_{1}, \ldots, c_{n-1} \in C_{n-1}$ are such that $\left(c_{1}, \ldots, c_{n-1}\right)$ is a free tuple. Then there is a representative $c_{n} \in C_{n}$ such that the tuple $\left(c_{1}, \ldots, c_{n}\right)$ is free.

Proof. Suppose that $c_{1}, \ldots, c_{n-1}$ are given and we have to construct $c_{n}$. Fix some $c \in C_{n}$. We seek $g \in \mathrm{PSL}_{m}(K)$ such that the $n$-tuple $\left(c_{1}, \ldots, c_{n}\right)$ is free with $c_{n}=g^{-1} c g$. We will first find out $g$ in a larger group $G(\Omega)=\operatorname{PSL}_{m}(\Omega)$ and then apply Borel's lemma. Let $W$ be the set of all non-empty words $w\left(x_{1}, \ldots, x_{n}\right)$ which are reduced on the tuple $\left(c_{1}, \ldots, c_{n-1}, c\right)$. Thus $W=\left\{w=w\left(x_{1}, \ldots, x_{n}\right)\right.$ : $w$ is reduced, $x_{i}^{m}$ does not occur in $w$ when $|m| \geq\left|c_{i}\right|$ and $x_{n}^{m}$ does not occur in $w$ when $\left.|m| \geq|c|\right\}$.

Let $w=w\left(x_{1}, \ldots, x_{n}\right) \in W$. The replacement $x_{1} \mapsto c_{1}, \ldots, x_{n-1} \mapsto c_{n-1}, x_{n} \mapsto x^{-1} c x$ gives rise to the monomial $w\left(c_{1}, \ldots, c_{n-1}, x^{-1} c x\right)$ over $G$ in variable $x$, which is not necessarily reduced over $G$. Indeed, for every maximal occurrence of $x_{n}^{m}$ in $w$ the result of substitution $x_{n} \mapsto x^{-1} c x$ contains a monomial $\left(x^{-1} c x\right)^{m}$ which is clearly not reduced. The replacement $\left(x^{-1} c x\right)^{m}$ by $x^{-1} c^{m} x$ is a non-empty monomial $w\left(c_{1}, \ldots, c_{n-1}, x^{-1} c x\right)_{r e d}$ with variable $x$ over $G$ and this monomial is reduced over $G$. The set

$$
\begin{aligned}
G(w) & =\left\{g \in G(\Omega): w\left(c_{1}, \ldots, c_{n-1}, g^{-1} c g\right)=1\right\} \\
& =\left\{g \in G(\Omega): w\left(c_{1}, \ldots, c_{n-1}, g^{-1} c g\right)_{r e d}=1\right\}
\end{aligned}
$$

is an algebraic subset of $G(\Omega)$ given by $w\left(c_{1}, \ldots, c_{n-1}, x^{-1} c x\right)_{\text {red }}=1$. It is defined over the field $L=$ $k\left(c_{1}, \ldots, c_{n-1}, c\right)$ which is finitely generated over the prime subfield $k$ and contains the field of definition $k$ of $G$. The irreducible components $G(w)_{1}, \ldots, G(w)_{n_{w}}$ of $G(w)$ are defined over the separable closure $L_{s}$ Bo1, $\left.\S 12.3\right]$. Each $G(w)$ is a proper subset of $G(\Omega)$ since otherwise $w\left(c_{1}, \ldots, c_{n-1}, x^{-1} c x\right)_{\text {red }}=1$ is a non-empty generalized law on $G=\operatorname{PSL}_{m}(\Omega)$, which contradicts Theorem 31. The family of algebraic subsets $\left\{G(w)_{i}: w \in W\right\}$ is countable. Finally, the group $G$, being reductive, is unirational over $k$ Bo2, section 18.2]. All above means that we can apply Borel's lemma 32 with $X=G, L=k\left(c_{1}, \ldots, c_{n-1}, c\right)$ and $\left(V_{i}\right)$ the family of all irreducible components of all $G(w), w \in W$.

We conclude that $G(K)$ is not contained in the union $\bigcup_{w \in W} G(w)$. The complement

$$
G(\Omega)-\bigcup_{w \in W} G(w)
$$

consists of $g \in G(\Omega)$ such that the $n$-tuple $\left(c_{1}, \ldots, c_{n-1}, g^{-1} c g\right)$ is free. Hence $G(K)$ contains a point $g$ outside of union $\bigcup_{w \in W} G(w)$, so the corresponding tuple $\left(c_{1}, \ldots, c_{n-1}, g^{-1} c g\right)$ is free and $K$-rational.

\subsection{When is $\operatorname{PSL}_{m}(K)$ an $\mathcal{I G}$ ?}

Theorem 34 If a field $K$ is a countable and has infinite transcendence degree over the prime field, then the group $\mathrm{PSL}_{m}(K)$ is not $\mathcal{I G}$ for $m \geq 2$. 
Proof. The group $G=\mathrm{PSL}_{m}(K)$ is countable. Then $G-\{e\}=\cup C_{i},(i \in \mathbb{N})$, where $C_{i}$ are all non-trivial distinct conjugacy classes. Inductively applying Lemma 33 we find out representatives $c_{i}$ so that the subgroup $\left\langle c_{i}: i \in \mathbb{N}\right\rangle$ is isomorphic to the free product $C=*\left\{c_{i}^{\mathbb{Z}}: i \in \mathbb{N}\right\}$. By construction, $C$ meets all conjugacy classes and is non-trivial. The set $S=\left\{c_{i}\right\}$ is conjugation complete. Moreover, $\left\langle c_{i}: i \in \mathbb{N}\right\rangle$ is a proper subgroup of $G$ because its abelianization is $C^{a b}=\prod_{i}\left(\mathbb{Z} /\left|c_{i}\right|\right)^{\mathbb{N}}$ (where $\left|c_{i}\right|=0$ if $c_{i}$ is of infinite order ) is non-trivial whereas $G$ is a perfect group, even simple.

Theorem 35 There are uncountably many isomorphism types of non-IG groups of the form $\operatorname{PSL}_{m}(K)$, where $m \geq 2$ and $K$ is a countable field of infinite transcendence degree over $\mathbb{Q}$.

Proof. Let $P$ denote the set of all primes in $\mathbb{N}$ and let $R \subseteq P$ be any subset. Consider the fields $\mathbb{Q}(\sqrt{R})$ generated by square roots of all the primes in $R$. Each of these fields is countable, and it's not too hard to see that they are pairwise non-isomorphic. Now adjoin to each $\mathbb{Q}(\sqrt{R})$ countably many indeterminates $X=\left\{X_{i}: i \in \mathbb{N}\right\}$, i.e. consider the field of rational functions $\mathbb{Q}(\sqrt{R}, X)$ in indeterminates $X$. The fields $\mathbb{Q}(\sqrt{R}, X)$ are all countable, pairwise non-isomorphic and have infinite transcendence degree over the prime field $\mathbb{Q}$.

By the result of Schreier and van der Waerden [SW] the groups $\operatorname{PSL}_{m}(K), \operatorname{PSL}_{m^{\prime}}\left(K^{\prime}\right),\left(m, m^{\prime} \geq 2\right)$ can be isomorphic only when $m=m^{\prime}$, excluding the case of $\mathrm{PSL}_{2}\left(\mathbb{F}_{7}\right) \simeq \mathrm{PSL}_{3}\left(\mathbb{F}_{2}\right)$. Furthermore, if $m=m^{\prime}>2$, the isomorphism is possible only when the fields $K, K^{\prime}$ are isomorphic. The same is true when $m=m^{\prime}=2$, excluding the case $\left\{K, K^{\prime}\right\}=\left\{\mathbb{F}_{4}, \mathbb{F}_{5}\right\}$. We conclude that the groups $\operatorname{PSL}_{\mathrm{m}}(\mathbb{Q}(\sqrt{\mathrm{R}}, \mathrm{X})), m \geq 2, R \subseteq P$, are pairwise non-isomorphic.

Similar construction can be carried over when the prime field is finite.

\section{Open problems}

There are several open problems, concerning $\mathcal{I} \mathcal{G}$.

1. : Is an infinite compact group $\mathcal{T} \mathcal{I G}$ if and only if it is connected abelian by profinite KLS2 ?

2. : $\mathrm{Is}_{n}(\mathbb{Z}),(n \geq 3) \mathcal{I} \mathcal{G}$ KLS2]?

3. : $\operatorname{Is~}_{\mathrm{SL}_{n}}(\mathbb{Q}),(n \geq 2) \mathcal{I G}[\mathrm{KLS2}$ ?

4. : Is $\mathrm{SL}_{2}(\mathbb{R})$, viewed as an abstract group, $\mathcal{I G}$ (Gelander)?

5. : Is every $\mathcal{I G}$-linear group virtually solvable KLS2]

6. Is a finite index subgroup of an $\mathcal{I G}$ group necessarily $\mathcal{I G}$ (Wiegold)? According to the referee's advise this can be generalized as follows: Is a lattice in a $\mathcal{T} \mathcal{I G}$ group an $\mathcal{I G}$ group? Regretfully the 
answer is negative - as we proved $\mathrm{SL}_{2}(\mathbb{R})$ is $\mathcal{T} \mathcal{I G}$ but contains a cocompact discrete group which is $\mathcal{I G}$ by Gelander's result. It would be nice to describe Lie groups and their lattices for which the above question has a positive answer.

7. "I think it is worth noting that every group outside $\mathcal{I} \mathcal{G}$ that I know satisfies no non-trivial law, and it would be nice to have an example with a non-trivial law. For example, can a Tarski like group of finite exponent be outside $\mathcal{I} \mathcal{G}$ ?" Wi2 .

\section{Acknowledgements}

Acknowledgements 36 This paper was inspired by numerous discussions within the Midrasha seminar at the Weizmann's Institute of Science, which we admirably acknowledge. The research was supported by the Weizmann Institute of Science, SFB 701 of Bielefeld University and the program of fundamental scientific research of the SB RAS I.1.1., project 0314-2019-0004.

Both authors are thankful to the referee for constructive comments and recommendations.

\section{References}

[Ad] J. F. Adams, Lectures on Lie groups, University of Chicago Press, 1982.

[Ax] S. J. Axler, Linear algebra done right, Vol. 2. New York: Springer, 1997.

[BORT] H. Bass, M. V. Otero-Espinar, D. Rockmore and C. Tresser, Cyclic renormalization and automorphism groups of rooted trees, Lecture Notes in Mathematics, Volume 1621, Springer, 1996.

[Be] A. Berarducci, Zero-Groups And Maximal Tori, in: A. Andretta, K. Kearnes, D. Zambella (Eds.), Logic Colloquium 04, in: Lecture Notes in Logic 29 (2006), pages 3347

[BM] M. Burger and S. Mozes, Groups acting on trees: from local to global structure, Publications Mathmatiques de l'Institut des Hautes tudes Scientifiques 92.1 (2000), pages 113-150.

[Bo1] A. Borel, On free subgroups of semisimple groups, Enseignement Mathematique (2) 29 (1983), pages 151-164. FIX

[Bo2] A. Borel, Linear algebraic groups, Vol. 126. New York: Springer, 1991.

[Ch] N. Tschebotareff, Die Bestimmung der Dichtigkeit einer Menge von Primzahlen, welche zu einer gegebenen Substitutionsklasse gehoren, Mathematische Annalen 95.1 (1926), pages 191-228.

[Co] K. Conrad, Decomposing $\mathrm{SL}_{2}(\mathbb{R})$, http://www.math.uconn.edu/kconrad/blurbs/grouptheory/SL(2, R).pdf. 
[Co] B. Conrad, Reductive groups over fields (version of august 11, 2017), Lectures by Brian Conrad, notes by Tony Feng, http://math.stanford.edu/ conrad/249BW16Page/handouts/249B_2016.pdf.

[Di] J. D. Dixon, Random sets which invariably generate the symmetric group, Discrete mathematics 105.1-3 (1992), pages 25-39.

[DGHV] J. J. Duistermaat, V. W. Guillemin, L. Hormander and D. Vassiliev, Fourier integral operators, Vol. 2. Boston: Birkhuser, 1996.

[Ge] T. Gelander, Convergence groups are not invariably generated, International Mathematics Research Notices 2015.19 (2014), pages 9806-9814.

[GM] T. Gelander and C. Meiri, The Congruence Subgroup Property does not imply Invariable Generation, International Mathematics Research Notices 2017.15 (2016), pages 4625-4638.

[GGJ] T. Gelander, G. Golan and K. Juschenko, Invariable generation of Thompson groups, Journal of Algebra 478 (2017), pages 261-270.

[Gl] S. Glasner, Proximal flows, Springer, Berlin, Heidelberg, 1976, pages 17-29.

[GNS] P. Gawron, V. V. Nekrashevich and V. I. Sushchanskii, Conjugacy Classes of the Automorphism Group of a Tree, Mathematical Notes 65.6 (1999), pages 787-790.

[GM] I. Z. Golubchik and A. V. Mikhalev, Generalized group identities in classical groups, Journal of Mathematical Sciences 27.4 (1984), pages 2902-2918.

[Ja] N. Jacobson, Lie algebras, Interscience Tracts on Pure and. Applied Mathematics, no. 10. Interscience Publishers, New York, 1962. pages 9+331.

[Jo] C. Jordan, Recherches sur les substitutions, Journal de Mathmatiques Pures et Appliques 17 (1872), pages 351-367.

[KLS1] W. M. Kantor, A. Lubotzky and A. Shalev, Invariable generation and the Chebotarev invariant of a finite group, Journal of Algebra 348.1 (2011), pages 302-314.

[KLS2] W. M. Kantor, A. Lubotzky and A. Shalev, Invariable generation of infinite groups Journal of Algebra 421 (2015), pages 296-310.

[PKM] P. K. Suetin, A. I. Kostrikin and Y. I. Manin, Linear Algebra and Geometry, (Algebra, Logic and Applications), CRC Press, 1989. 
[KZ] E. Kowalski and D. Zywina, The Chebotarev invariant of a finite group, Experimental Mathematics 21.1 (2012), pages 38-56.

[Lu1] A. Lucchini, The Chebotarev invariant of a finite group: A conjecture of Kowalski and Zywina, Proceedings of the American Mathematical Society 146.11 (2018), pages 4549-4562.

[Lu2] A. Lucchini and G. Tracey, An upper bound on the Chebotarev invariant of a finite group Israel Journal of Mathematics 219.1 (2017), pages 449-467.

[La] S. Lang, Algebra (graduate texts in mathematics), Berlin, itd: Springer Verlag 211 (2002).

[Os] D. Osin, Small cancellations over relatively hyperbolic groups and embedding theorems, Annals of mathematics (2010), pages 1-39.

[PS] Y. Peterzil and C. Steinhorn, Definable compactness and definable subgroups of O-minimal groups, Journal of the London Mathematical Society 59.3 (1999), pages 769-786.

[Se] J. P. Serre, On a theorem Of Jordan, Bulletin of the American Mathematical Society 40.4 (2003), pages $429-440$.

[SW] O. Schreier and B. L. van der Waerden, Die Automorphismen der projektiven Gruppen, Abhandlungen aus dem Mathematischen Seminar der Universitt Hamburg. Vol. 6. No. 1. Springer-Verlag, 1928, pages 303-322.

[St] R. Steinberg, Endomorphisms of linear algebraic groups, No. 80. American Mathematical Soc., 1968.

[St] A. Strzebonski, Euler characteristic in semialgebraic and other O-minimal structures, J. Pure Appl. Algebra 96.2 (1994), pages 173-201.

[Ti] J. Tits, Sur le groupe des automorphismes d'un arbre, Essays on topology and related topics. Springer, Berlin, Heidelberg, 1970. pages 188-211.

[Wi1] J. Wiegold, Transitive groups with fixed-point-free permutations, Archiv der Mathematik 27.1 (1976), pages 473-475.

[Wi2] J. Wiegold, Transitive groups with fixed-point-free permutations.II, Archiv der Mathematik 29.1 (1977), pages 571-573. 